\title{
Evaluation of the Average Nasal and Nasopharyngeal Volume in 10-13-year-old Children: A Preliminary CBCT Study
}

\author{
Ritesh Kalaskar ${ }^{1}$, Shruti Balasubramanian², Ashita Kalaskar ${ }^{3}$
}

\begin{abstract}
Aim and objective: Mouth breathing is one of the most common deleterious habits prevalent in children which leads to various skeletal and dental malocclusions. Due to the close relationship between nasal and nasopharyngeal cavity volume and maxilla, transverse maxillary deficiency causes reduced nasal and nasopharyngeal cavity volume leading to mouth breathing. Therefore, knowledge of average nasal and nasopharyngeal cavity volume is essential to accurately diagnose mouth breathing and to evaluate underlying causative factors.

Materials and methods: Cone-beam computed tomographic scans of 60 children were taken and nasal cavity and nasopharyngeal volumes were calculated using Planmeca Romexis 5.2.0.R software. Average volumes were computed using predetermined landmarks and compared among gender.

Results: The nasal cavity and nasopharyngeal volume showed significant differences among the gender ( $p$ value $<0.001$ and 0.018 , respectively). Conclusion and clinical significance: Knowledge of the average nasal and nasopharyngeal cavity volumes can be a useful diagnostic aid for mouth breathing patients and also assess the causative factors and treatment outcomes in these patients.

Keywords: Cone-beam computed tomography, Cross-sectional study, Mouth breathing, Nasal cavity volume, Nasopharyngeal volume.

International Journal of Clinical Pediatric Dentistry (2021): 10.5005/jp-journals-10005-1917
\end{abstract}

\section{INTRODUCTION}

The upper airway principally consists of nasal and pharyngeal parts which influence the development of craniofacial structures. There exists an intimate anatomical relationship between the nasal and pharyngeal cavity volume and the maxilla due to which transverse deficiency in maxilla leads to reduced nasal and pharyngeal volume. Mouth breathing syndrome (MBS) is defined as a set of signs and symptoms that may be completely or incompletely present in subjects who, for various reasons, replace the correct pattern of nasal breathing with an oral or mixed pattern. ${ }^{1}$ It has been observed that mouth breathing persists even after correction of primary predisposing factors for mouth breathing such as the deviated nasal septum, obstructive adenoids, and chronic inflammation of nasal mucosa. ${ }^{2}$ Recent research on mouth breathing and rapid maxillary expansion (RME) had suggested transverse maxillary deficiency could be a primary cause of mouth breathing. . $^{2,3}$

Rapid maxillary expansion has been used for the correction of transverse maxillary deficiency which in turn leads to increased nasal and pharyngeal cavity volume. ${ }^{3,4}$ It is expected that increased nasal and pharyngeal volume increases nasal airflow which might alleviate mouth breathing. However, due to the lack of normal parameters on nasal and pharyngeal cavity volume, the effect of RME on mouth breathing could not be evaluated. Similarly, literature also lacks knowledge of whether all patients of transverse maxillary deficiency have reduced nasal and pharyngeal cavity volume. Therefore, knowledge of average nasal and pharyngeal cavity volume is essential to accurately diagnose mouth breathing and to evaluate underlying causative factors before the intervention, since it is known to affect the treatment modality.

Therefore, this preliminary study has been planned to evaluate the average nasal and nasopharyngeal volume in 10-13-year-old children using cone-beam computed tomography (CBCT) and to establish its significance as a diagnostic tool for mouth breathing.
1,2 Department of Pediatric and Preventive Dentistry, Government Dental College and Hospital, Medical College Premises, Medical Square, Nagpur, Maharashtra, India

${ }^{3}$ Department of Oral Medicine and Radiology, Government Dental College and Hospital, Medical College Premises, Medical Square, Nagpur, Maharashtra, India

Corresponding Author: Shruti Balasubramanian, Department of Pediatric and Preventive Dentistry, Government Dental College and Hospital, Medical College Premises, Medical Square, Nagpur, Maharashtra, India, Phone: +91 9535401205, e-mail: shruti.310194@ gmail.com

How to cite this article: Kalaskar R, Balasubramanian S, Kalaskar A. Evaluation of the Average Nasal and Nasopharyngeal Volume in 10-13-year-old Children: A Preliminary CBCT Study. Int J Clin Pediatr Dent 2021;14(2):187-191.

Source of support: Nil

Conflict of interest: None

\section{Materials and Methods}

After obtaining approval from the Institutional Ethical committee, this cross-sectional study was conducted on 60 children ( 36 males and 24 females) of age ranging from 10 to 13 years. All the children were exposed to CBCT and full-face scans were obtained. Male and female children without any relevant medical history were included in the study. Children with a history of adenotonsillar pathology, sinusitis, allergic rhinitis, mouth breathing, moderate to severe deviated nasal septum, intranasal tumor, and polyps, midfacial trauma, anterior and posterior crossbite were excluded from the study.

The Digital Imaging Communication in Medicine (DICOM) images of patients were obtained and the nasal cavity and nasopharyngeal volumes were calculated using the Planmeca 
Romexis 5.2.0.R (Fig. 1). The nasal cavity volume was calculated using certain predetermined landmarks by which the nasal cavity was delineated and the paranasal sinuses were excluded. This was done using the manual segmentation tool in the Planmeca Romexis 5.2.0.R software in the midsagittal section (MSP). In this method, the midsagittal section was divided into 24 sections of $0.4 \mathrm{~mm}$ thickness for obtaining accurate measurement. The manual segmentation tool was used to mark the boundaries of the nasal cavity in these sections from the anterior to the posterior portion of the sagittal section (Fig. 2). The anterior limit was the line that connects point $\mathrm{N}$ to point anterior nasal spine (ANS), the posterior limit was a line that connects the inferior border of sphenoid sinus (IBS) to the posterior nasal spine (PNS) and the inferior limit was a line that connects the ANS to PNS. The superior limit was marked manually, extending anteriorly till point $\mathrm{N}$ and posteriorly up to the IBS in the form of a curved line to ensure exclusion of the paranasal sinuses namely sphenoid, ethmoid, and maxillary sinus (Table 1 and Fig. 3).

Nasopharyngeal volume was calculated using predetermined landmarks ${ }^{5}$ in the midsagittal plane (Table 1) and the volume was calculated using the ellipsoid tool, thereafter airway space was delineated using the airway tool of the software (Fig. 3).

\section{Statistical Analysis}

Statistical analysis was performed using SPSS 16.0 software (IBM Corp., Armonk, N.Y., USA). The average nasal cavity and nasopharyngeal volume were calculated separately for males and females and expressed in mean \pm standard deviation (SD) and volumes expressed in $\mathrm{mm}^{3}$. An independent $t$-test was used to compare the volume between the gender and $p$ value $<0.05$ was considered to be statistically significant. All significant differences were expressed as $95 \%$ confidence intervals ( $95 \% \mathrm{Cl}$; $\alpha$ equals 0.05 ).

\section{Results}

The CBCT scans of 60 children comprising of 36 males and 24 females were analyzed to calculate the average nasal cavity and nasopharyngeal volume (Table 2). The average nasal cavity volume was $26,867.90 \pm 2,842.72 \mathrm{~mm}^{3}$ among males and 22,978.70 \pm $2,609.15 \mathrm{~mm}^{3}$ among females. It showed a significant difference among the gender ( $p$ value $<0.001$ ). The nasopharyngeal volume was computed to be $2,930.00 \pm 460.31 \mathrm{~mm}^{3}$ in males and 2,644.20 \pm $420.67 \mathrm{~mm}^{3}$ in females which also showed a statistically significant difference $(p$ value $=0.018$ ) (Table 3$)$.

\section{Discussion}

In the literature, studies evaluating the average nasal cavity and nasopharyngeal volume using $\mathrm{CBCT}$ are very infrequent. Various methods have been used in literature to evaluate the nasal and nasopharyngeal volume, namely lateral and posteroanterior (PA) cephalometry, rhinomanometry, acoustic rhinometry, computed tomography (CT), CBCT. ${ }^{6-9}$ Lateral and PA cephalometry being a 2D representation of 3D structures show superimposition, projection errors, and artifacts. Cankurtaran et al..$^{10}$ established that acoustic rhinometry is ineffective in calculating the volume of the posterior part of the nasal cavity, which was possible with CBCT especially in the midsagittal plane which was used in this study. Other methods of measurement, rhinomanometry, and CT have the disadvantage of being invasive and high radiation exposure, respectively, and also not readily available for dentists. Doruk et al. ${ }^{11}$ showed a good correlation between acoustic rhinometry and CT for analyzing nasal volume changes post RME. Tsolakis et al. ${ }^{12}$ compared the results of CBCT and acoustic rhinometry and concluded CBCT to be an accurate method for upper airway analysis. Therefore, CBCT has been considered one of the most precise methods for evaluating upper airway as it permits three-dimensional reconstruction with good reproducibility, reliability, and low radiation exposure. ${ }^{13-15}$

Calculation of nasal cavity volume is complex due to its intimate association with the paranasal sinuses namely maxillary, sphenoid, and ethmoid sinus. Various studies have been conducted in the literature for the calculation of nasal cavity volume using threedimensional imaging techniques. Yokley ${ }^{16}$ in an attempt to establish a correlation between the nasal index and nasal cavity volume divided the nasal passage into segments and used perimeter/ area ratio as a proxy to the surface area/volume ratio using CT. This method failed to achieve the accurate measurement of nasal cavity volume due to the use of proxy measurements. $\mathrm{Ha}$ and $\mathrm{Han}^{17}$ tried to calculate the volume of nasal cavity between anterior and

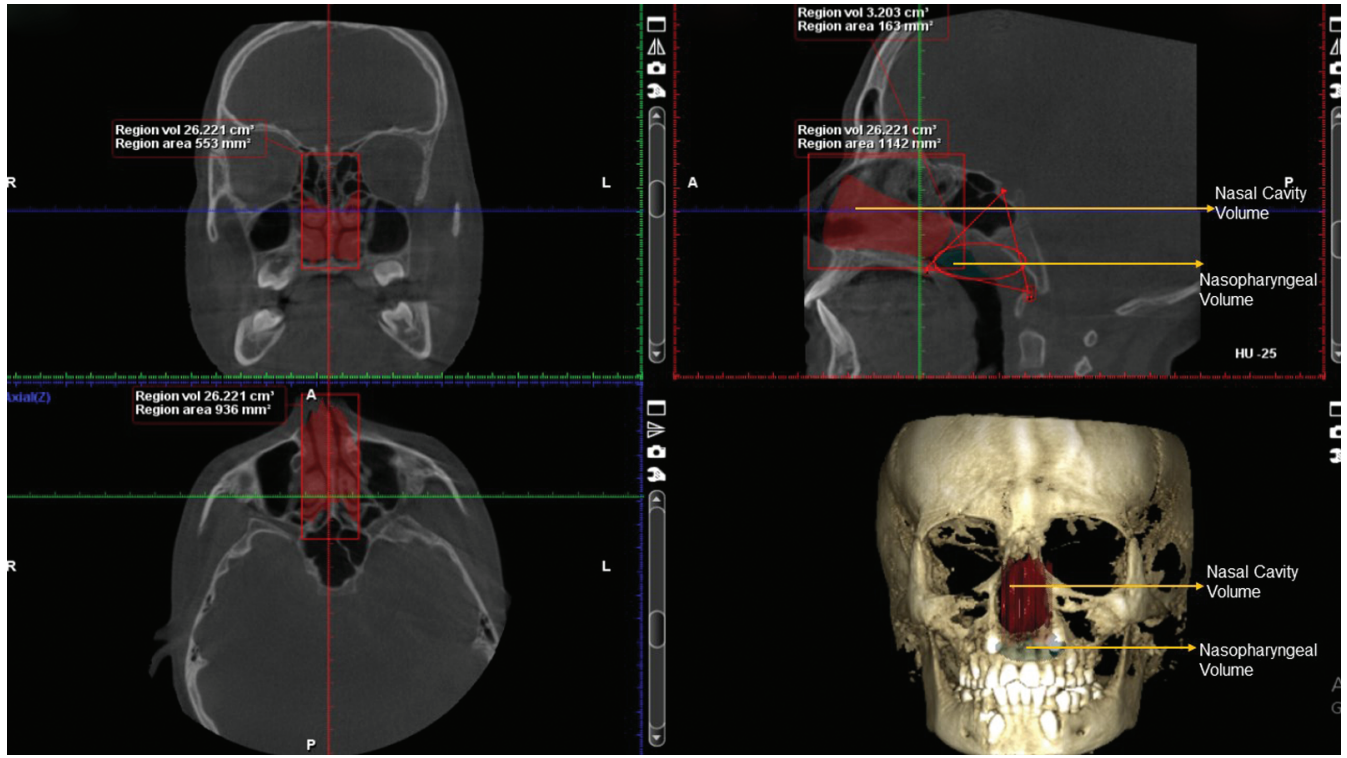

Fig. 1: Nasal cavity and nasopharyngeal volume in coronal, sagittal, axial, and three-dimensional view of Planmeca Romexis 5.2.0.R software 


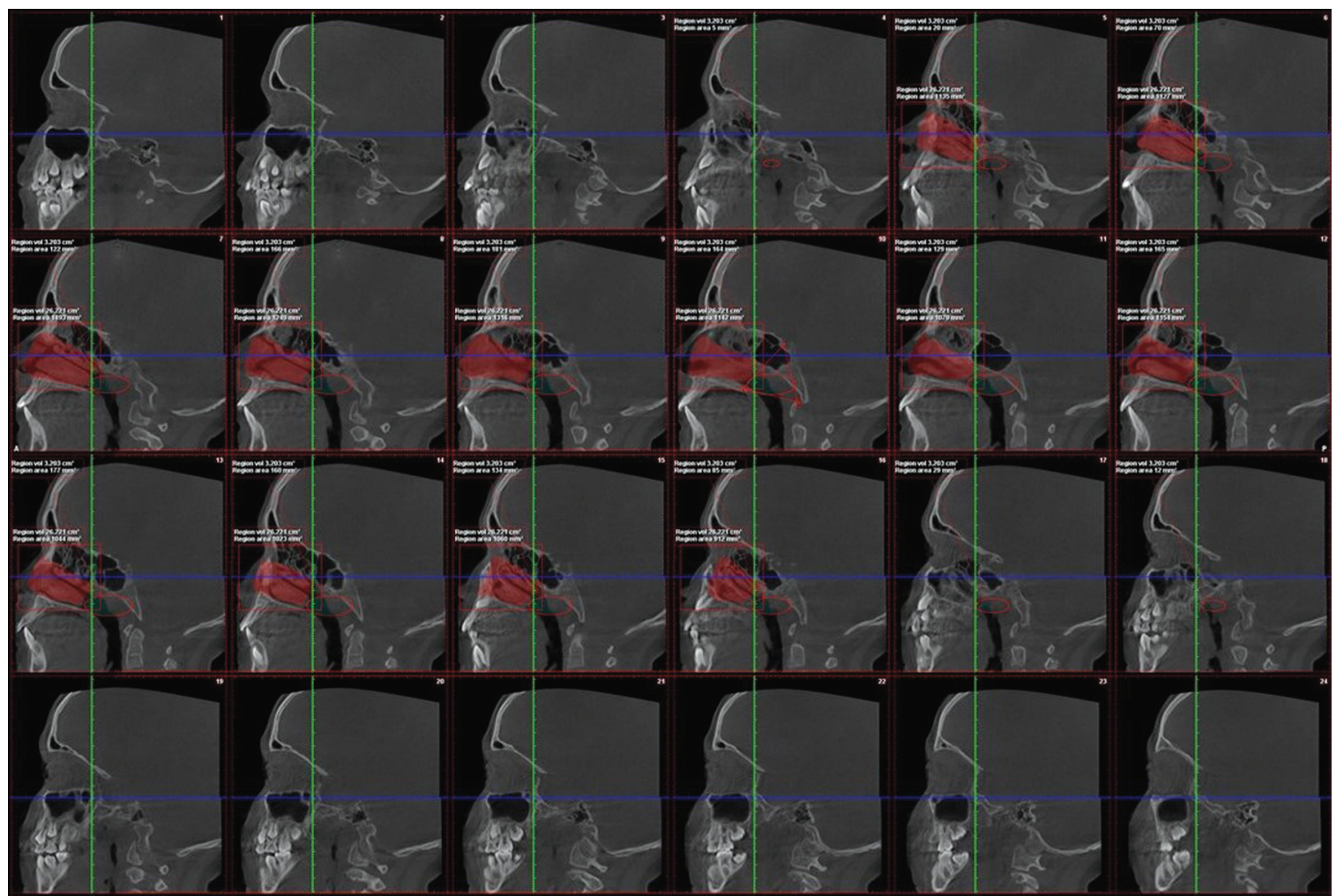

Fig. 2: Midsagittal section divided into 24 sections of $0.4 \mathrm{~mm}$ thickness each to calculate nasal cavity volume using manual segmentation tool

Table 1: CBCT landmarks for nasal cavity and nasopharyngeal volume

\begin{tabular}{lllll}
\hline Area & Anterior limit & Posterior limit & Superior limit & Inferior limit \\
\hline Nasal cavity & $\begin{array}{l}\text { Line that connects } \\
\text { point N to ANS, in } \\
\text { the MSP }\end{array}$ & $\begin{array}{l}\text { Line that connects } \\
\text { IBS to the PNS, in } \\
\text { the MSP }\end{array}$ & $\begin{array}{l}\text { It was marked manually, extending anteriorly till point N } \\
\text { and posteriorly up to the IBS in the form of a curved line } \\
\text { to ensure exclusion of the paranasal sinuses }\end{array}$ & $\begin{array}{l}\text { Line that connects } \\
\text { the ANS to PNS, in } \\
\text { the MSP }\end{array}$ \\
& $\begin{array}{l}\text { Line that connects } \\
\text { the PNS to point S, }\end{array}$ & $\begin{array}{l}\text { Line that connects } \\
\text { point S to the SPA, }\end{array}$ & - & Line that connects \\
in the MSP & in the MSP & & the SPA to the PNS, \\
& in the MSP
\end{tabular}

*MSP, midsagittal plane; ANS, anterior nasal spine; IBS, inferior border of sphenoid sinus; PNS, posterior nasal spine; SPA, more superior point on the atlas

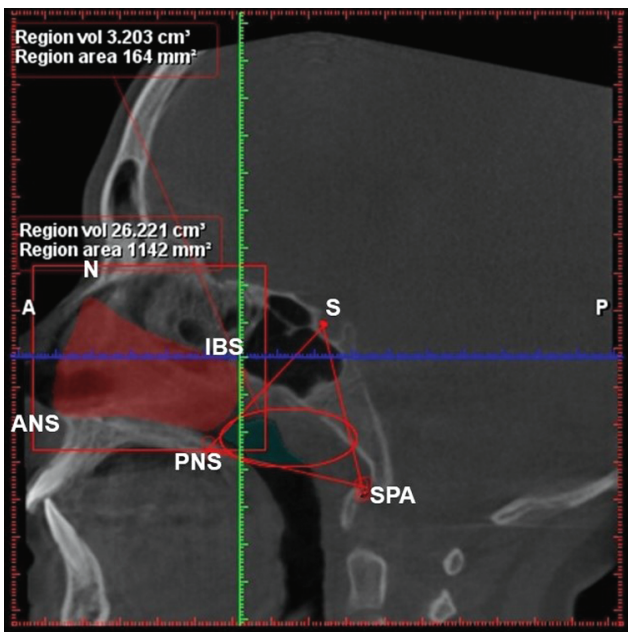

Fig. 3: Landmarks for calculation of nasal and nasopharyngeal volume in the midsagittal plane posterior nares excluding frontal, ethmoid, and maxillary sinus. They used multidetector computed tomographic (MDCT) images and Mimics software. Thereafter, post the advent of CBCT upper airway analysis was being attempted with even greater accuracy. Martins et al. ${ }^{18}$ attempted to establish a correlation between 2D and 3D imaging for upper airway analysis measured nasal cavity volume using Dolphin imaging. The anterior limit was the ANS line through up to nasal bone and the posterior limit was the PNS line up to skull base. The nasal cavity volume was then calculated using the airway space tool. The effect of surgical-assisted RME on upper airway volume was analyzed by Romulo de Medeiros et al. ${ }^{5}$ using Dolphin 3D software. They defined the limits of the nasal cavity as the anterior limit being the line joining point $N$ to point $A$, posterior limit extending from point $S$ to PNS, superior limit extending from point $\mathrm{N}$ to point $\mathrm{S}$, and lastly inferior limit from ANS to point $\mathrm{S}$ in the midsagittal plane. However, these landmarks failed to exclude the paranasal sinuses. A systematic review conducted by Buck et al. ${ }^{19}$ reported a great diversity in the methodologies used for upper airway volume calculation which generated an inherent 
difficulty in comparing results between studies. To standardize the anatomic limits of the nasal cavity, modifications were made in the landmarks given by Romulo de .. ledeiros et al. To ensure exclusion of paranasal sinuses namely sphenoid, ethmoid and maxillary sinus, and delineate the nasal cavity, a manual segmentation tool was used to manually mark the region of interest.

Table 2: Nasal cavity and nasopharyngeal volume of males and females

\begin{tabular}{|c|c|c|c|}
\hline \multicolumn{2}{|c|}{ Nasal cavity volume $\left(\mathrm{cm}^{3}\right)$} & \multicolumn{2}{|c|}{ Nasopharyngeal volume $\left(\mathrm{cm}^{3}\right)$} \\
\hline Male & Female & Male & Female \\
\hline 28.215 & 19.78 & 2.001 & 2.264 \\
\hline 26.221 & 29.321 & 3.203 & 3.184 \\
\hline 29.552 & 20.938 & 3.083 & 2.866 \\
\hline 23.963 & 27.511 & 3.01 & 2.782 \\
\hline 19.711 & 21.733 & 3.173 & 3.367 \\
\hline 30.469 & 19.708 & 2.464 & 2.715 \\
\hline 21.51 & 27.047 & 2.281 & 2.225 \\
\hline 27.79 & 21.206 & 3.223 & 3.464 \\
\hline 21.384 & 19.107 & 3.621 & 3.6 \\
\hline 25.323 & 21.108 & 2.325 & 2.183 \\
\hline 28.36 & 22.708 & 2.44 & 3.115 \\
\hline 21.5 & 24.093 & 3.622 & 2.854 \\
\hline 24.67 & 22.898 & 2.108 & 2.282 \\
\hline 26.302 & 21.8 & 2.564 & 2.387 \\
\hline 27.371 & 23.768 & 2.944 & 2.415 \\
\hline 21.325 & 19.708 & 3.348 & 2.223 \\
\hline 26.908 & 21.345 & 2.355 & 2.322 \\
\hline 26.52 & 25.678 & 3.556 & 2.41 \\
\hline 27.85 & 22.65 & 3.45 & 2.465 \\
\hline 28.805 & 23.568 & 3.65 & 2.469 \\
\hline 25.534 & 24.76 & 3.001 & 2.512 \\
\hline 28.464 & 22.678 & 2.677 & 2.456 \\
\hline 27.89 & 23.769 & 3.114 & 2.345 \\
\hline 25.677 & 24.607 & 3.29 & 2.556 \\
\hline 28.607 & & 2.73 & \\
\hline 29.351 & & 3.312 & \\
\hline 30.521 & & 3.098 & \\
\hline 28.56 & & 2.416 & \\
\hline 27.286 & & 2.934 & \\
\hline 30.257 & & 3.433 & \\
\hline 28.576 & & 2.545 & \\
\hline 28.567 & & 2.584 & \\
\hline 26.668 & & 3.21 & \\
\hline 28.879 & & 3.244 & \\
\hline 29.998 & & 2.983 & \\
\hline 28.66 & & 2.488 & \\
\hline
\end{tabular}

Table 3: Average nasal cavity and nasopharyngeal volume $\left(\mathrm{mm}^{3}\right)$

\begin{tabular}{lllll}
\hline & Gender & Mean $\pm S D\left(\mathrm{~mm}^{3}\right)$ & $p$ value & $95 \% \mathrm{Cl}$ \\
\hline Nasal cavity volume & Male & $26,867.90 \pm 2,842.72$ & $<0.001^{*}$ & $25,906.00-27,829.70$ \\
& Female & $22,978.70 \pm 2,609.15$ & $21,877.00-24,080.50$ & $2,774.30-3,085.70$ \\
Nasopharyngeal volume & Male & $2,930.00 \pm 460.31$ & $0.018^{*}$ & $2,466.60-2,821.80$ \\
& Female & $2,644.20 \pm 420.67$ & & 2,8
\end{tabular}

*Statistically significant $(p<0.05)$

$\mathrm{Cl}$, confidence interval; SD, standard deviation
This research mainly focused on the calculation of the average nasal cavity and nasopharyngeal volume and its difference between the gender. In the current study, the average nasal cavity volume was $26,867.90 \pm 2,842.72 \mathrm{~mm}^{3}$ among males and 22,978.70 \pm $2,609.15 \mathrm{~mm}^{3}$ among females. It showed a significant difference among males and females $(p<0.001)$. This observation from the current study is in accordance with the reported literature worldwide. Ertekin et al. ${ }^{20}$ computed average nasal cavity volume using CT in different age groups. He observed a significant difference in nasal cavity volume among gender in the age group of 14-18 years. Emirzeoglu et al. ${ }^{21}$ documented larger nasal cavity volume in males as compared to females using a stereological method with coronal CT images. Similar observation using CBCT images was observed by Martins et al. ${ }^{18}$ nasal cavity volume among male as 15,073.98 $\pm 4,196.18$ and 13,714.43 $\pm 3,723.32 \mathrm{~mm}^{3}$ among females ( $p$ value $=0.007$ ). The values obtained by Martins et al. ${ }^{18}$ were less than the volume obtained in this research which may be attributed to the different landmarks used. Romulo de Medeiros et al. studied the nasal cavity volume in patients with transverse maxillary deficiency preoperatively and postoperatively after pterygomaxillary disjunction. The nasal cavity volume was $28,089.3$ $\pm 2,095.4$ and $27,096.4 \pm 1,413.4 \mathrm{~mm}^{3}$, respectively. These values are similar to the ones obtained in this study.

To the best of our knowledge, this is a pioneer prospective study evaluating the average nasopharyngeal volume using CBCT. Pharyngeal airway space dimensions are known to be closely related to the sagittal as well as vertical skeletal pattern. Therefore, the knowledge of average nasopharyngeal volume can also function as a crucial assessment parameter. The nasopharyngeal cavity volume was computed to be $2,930 \pm 460.31 \mathrm{~mm}^{3}$ in males and $2,644.20 \pm 420.67 \mathrm{~mm}^{3}$ in females. It also showed significant differences between the gender ( $p$ value $=0.018$ ). In contrast to the results of our study, Martins et al..$^{18}$ in their retrospective analysis did not obtain a significant difference between the gender. In his study, the anterior limit of the nasopharynx was the posterior limit of the nasal cavity and the inferior limit as the PNS line extending to the pharynx's posterior wall. The nasopharyngeal volume calculated among male was $8,663.71 \pm 2,617.74 \mathrm{~mm}^{3}$ and among females was $8,250.65 \pm 2,030.61 \mathrm{~mm}^{3}$. The significantly high volumes obtained in this study in contrast to our study are unclear and may be attributed to lack of clear specification of the landmarks which may have included portions of the nasal cavity also during computation. Also, the main disadvantage of retrospective analysis is that acute clinical conditions like sinusitis, allergic rhinitis at the time of examination cannot be assessed which may influence the nasal cavity volume. This was overcome in the current study since these patients were excluded. Romulo de Medeiros et al. ${ }^{5}$ calculated the nasopharyngeal volume $5,245.1 \pm 664.5 \mathrm{~mm}^{3}$ preoperatively and $6,043.2 \pm 666.8 \mathrm{~mm}^{3}$ after pterygomaxillary disjunction. Despite the use of the same landmarks as a reference in our study, a significant difference was seen in the resultant volumes. This may be attributed to the wide difference in the age group which were studied by 
Romulo de Medeiros et al., ${ }^{5}$ i.e., $18-45$ years, whereas in this study pediatric population was considered. Therefore, it is advocated to conduct more age-related studies to evaluate the average nasopharyngeal volume using these standardized landmarks.

Over the years, varying advancements have taken place in the treatment protocol for mouth breathing and maxillary constriction ranging from RME to nasal stimulator, oral obturator. ${ }^{22}$ However, despite these advances, there is a lack of an objective method for its accurate diagnosis. The main implication of this study is to establish the knowledge of average nasal and nasopharyngeal volume as a crucial diagnostic tool for mouth breathers. Therefore, if similar studies are carried out in a larger sample, the results can be extrapolated to the entire population in the future.

\section{Conclusion and Clinical Significance}

Based on the results of this study, the following conclusions can be made:

- The knowledge of the average nasal and nasopharyngeal cavity volume would function as an objective tool for diagnosis of mouth breathing patients in contrast to the currently existing subjective and perception-based methods of diagnosis.

- It can also be useful to evaluate various treatment modalities and to assess its effect in actually alleviating mouth breathing habits.

\section{References}

1. Conti PB, Sakano E, Ribeiro MA, et al. Assessment of the body posture of mouth-breathing children and adolescents. J Pediatr (Rio J) 2011;87(4):357-363. DOI: 10.2223/JPED.2102.

2. Cunha DA, Silva GAP, Motta MEFA, et al. Mouth breathing in children and its repercussions in the nutritional state. Rev Cefac 2007;9(1):4754. DOI: 10.1590/S1516-18462007000100007.

3. El H, Palomo JM. Three-dimensional evaluation of upper airway following rapid maxillary expansion: a CBCT study. Angle Orthod 2014;84(2):265-273. DOI: 10.2319/012313-71.1.

4. Smith T, Ghoneima A, Stewart K, et al. Three-dimensional computed tomography analysis of airway volume changes after rapid maxillary expansion. Am J Orthod Dentofacial Orthop 2012;141(5):618-626. DOI: 10.1016/j.ajodo.2011.12.017.

5. Romulo de Medeiros J, Ferraro Bezerra M, Gurgel Costa FW, et al. Does pterygomaxillary disjunction in surgically assisted rapid maxillary expansion influence upper airway volume? A prospective study using Dolphin imaging 3D. Int J Oral Maxillofac Surg 2017;46(9):1094-1101. DOI: 10.1016/j.ijom.2017.04.010.

6. Chung $\mathrm{CH}$, Font B. Skeletal and dental changes in the sagittal, vertical, and transverse dimensions after rapid palatal expansion. Am J Orthod Dentofacial Orthop 2004;126(5):569-575. DOI: 10.1016/ j.ajodo.2003.10.035.
7. Kurt G, Altug-Atac AT, Atac MS, et al. Changes in nasopharyngeal airway following orthopedic and surgically assisted rapid maxillary expansion. J Craniofac Surg 2010;21(2):312-317. DOI: 10.1097/ SCS.0b013e3181cf5f73.

8. Gordon JM, Rosenblatt M, Witmans M, et al. Rapid palatal expansion effects on nasal airway dimensions as measured by acoustic rhinometry. A systematic review. Angle Orthod 2009;79(5):1000-1007. DOI: 10.2319/082108-441.1.

9. Montgomery WM, Vig PS, Staab EV, et al. Computed tomography: a three-dimensional study of the nasal airway. Am J Orthod 1979;76(4):363-375. DOI: 10.1016/0002-9416(79)90223-9.

10. Cankurtaran $\mathrm{M}$, Çelik H, Çakmak Ö, et al. Effects of the nasal valve on acoustic rhinometry measurements: a model study. J Appl Physiol 1985 2003;94(6):2166-2172. DOI: 10.1152/japplphysiol.01146.2002.

11. Doruk C, Sokucu O, Bicakci AA, et al. Comparison of nasal volume changes during rapid maxillary expansion using acoustic rhinometry and computed tomography. Eur J Orthod 2007;29(3):251-255. DOI: 10.1093/ejo/cjl069.

12. Tsolakis IA, Venkat $D$, Hans MG, et al. When static meets dynamic: Comparing cone-beam computed tomography and acoustic reflection for upper airway analysis. Am J Orthod Dentofacial Orthop 2016;150(4):643-650. DOI: 10.1016/j.ajodo.2016.03.024.

13. Palomo JM, Rao PS, Hans MG. Influence of CBCT exposure conditions on radiation dose. Oral Surg Oral Med Oral Pathol Oral Radiol Endod 2008;105(6):773-782. DOI: 10.1016/j.tripleo.2007.12.019.

14. El H, Palomo JM. Measuring the airway in 3 dimensions: a reliability and accuracy study. Am J Orthod Dentofacial Orthop 2010;137(4 Suppl):S50.e1-9. DOI: 10.1016/j.ajodo.2010.01.014.

15. Osorio F, Perilla M, Doyle DJ, et al. Cone beam computed tomography: an innovative tool for airway assessment. Anesth Analg 2008;106(6):1803-1807. DOI: 10.1213/ane.0b013e318172fd03.

16. Yokley TR. Ecogeographic variation in human nasal passages. Am J Phys Anthropol 2009;138(1):11-22. DOI: 10.1002/ajpa.20893.

17. Ha YC, Han SJ. A 3-dimensional analysis of nasal cavity volume after maxillary Le Fort I osteotomy. J Oral Maxillofac Surg 2018;76(6):1344. e1-1344.e7. DOI: 10.1016/j.joms.2017.12.033.

18. Martins LS, Liedke GS, Heraldo LDS, et al. Airway volume analysis: is there a correlation between two and three-dimensions? Eur J Orthod 2018;40(3):262-267. DOI: 10.1093/ejo/cjx067.

19. Buck LM, Dalci O, Darendeliler MA, et al. The effect of surgically assisted rapid maxillary expansion (SARME) on upper airway volume: a systematic review. J Oral Maxillofac Surg 2016;74(5):1025-1043. DOI: 10.1016/j.joms.2015.11.035.

20. Ertekin T, Değermenci M, Nisari M, et al. Age-related changes of nasal cavity and conchae volumes and volume fractions in children: a stereological study. Folia Morphol 2016;75(1):38-47. DOI: 10.5603/ FM.a2015.0078.

21. Emirzeoglu M, Sahin B, Celebi M, et al. Estimation of nasal cavity and conchae volumes by stereological method. Folia Morphol 2012;71(2):105-108.

22. Denotti G, Ventura $S$, Arena $O$, et al. Oral breathing: new early treatment protocol. J Pediatr Neonat Individual Med 2014;3(1):e030108. 